\title{
Application of nonequilibrium concentration profiles in particle diffusion under the action of a magnetic force (abstract)
}

J. J. M. Janssen, H.W. Myron, and J. A. A. J. Perenboom

High Field Magnet Laboratory and Research Institute for Materials, NL-6525 ED Nijmegen,

The Netherlands

For submicron particles difusion effects and interparticle interactions cannot be neglected in studies of particle behaviour under the action of a magnetic force. Several authors have studied the case of single wire HGMS, where these effects lead to the phenomenon of dynamic buildup: a concentration gradient is induced, but no actual capture occurs. Applications are in, e.g.s magnetohydrodynamic separation ${ }^{2}$ and susceptibility selective separation. ${ }^{2}$ These appilications are based on the equilibrium situation. We have studied the case of nonequilibrium concentration through a mumerical solution of the time-dependent difusion equation, and have found interesting features, that may lead to new applications. The magnetic force will cause a depletion of (ferro) magnetic particles at one end of the sample cell at a size-dependent speed. Observation of the time evolution of the particle concentration at some distance from the end of the cell will then allow to deduce the particle size distribution.

'U. Andres, Minerals \$ci. Eng. 7, 99 (1975).

2J. Y. Hwang, M. Takayasu, F. J. Friedlaender, and G. Kullerud, J. Appi. Phys. 55, 2595 (1984). 